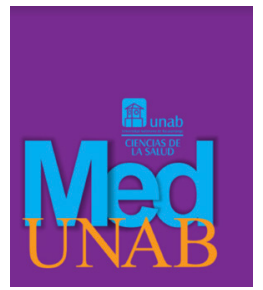

REVISTA DE LA FACULTAD

DE CIENCIAS DE LA SALUD

\title{
Erupción polimorfa durante el puerperio inmediato
}

Polymorphic eruption during the postpartum period

Erupção polimórfica durante o puerpério imediato

\section{Yelson Alejandro Picón Jaimes., MD. ${ }^{1}$, Javier Esteban Orozco Chinome., MD. ${ }^{2}$, Leonor Andrea Mejía Antolínez., MD., Esp ${ }^{3}$, Christi Tatiana Garcés Salamanca, MD. ${ }^{4}$}

1. Médico, Diploma en Salud Pública, Docente Universidad Pedro de Valdivia, Santiago, Chile.

2. Médico cirujano, Diploma en dermatología, Red Salud, Santiago, Chile.

3. Médica, Especialista en Administración de la Salud. Virrey Solís IPS, Bogotá, Colombia.

4. Médica, Residente de Ginecología y Obstetricia, Universidad Industrial de Santander, Bucaramanga, Colombia.

Correspondencia: Yelson Alejandro Picón Jaimes. Avenida Pocuro 2260, Providencia, Santiago, Chile. E-mail: ypicon@unab.edu.co

Cómo citar: Picón-Jaimes YA, Orozco-Chinome JE, Mejía-Antolínez LA, Garcés-Salamanca CT. Erupción polimorfa durante el puerperio inmediato. MedUNAB. 2019;22(1):64-70. doi:10.29375/01237047.3461

\section{INFORMACIÓN ARTÍCULO}

Artículo recibido: 28 de noviembre de 2018

Artículo aceptado: 07 de mayo de 2019

DOI: https://doi.org/10.29375/01237047.3461

\section{RESUMEN}

Introducción. En el embarazo, las dermatosis afectan a menos del $20 \%$ de las gestantes y representan un grupo heterogéneo de afecciones cutáneas, con una forma variada de presentación y evolución. El objetivo es presentar un caso de erupción polimorfa de inicio durante el puerperio, con una breve revisión de la literatura en torno a la patología y su tratamiento.

Reporte de caso. Se presenta el caso de una paciente de 38 años en puerperio de su primera gestación, quien debutó con un cuadro clínico de erupción cutánea y prurito que inició en la zona abdominal y se extendió hacia los miembros inferiores. Las lesiones estaban constituidas por pápulas eritematosas que confluían hasta formar 
placas. Se diagnosticó con erupción polimorfa del embarazo y se dio manejo con antihistamínicos logrando la resolución de la patología.

Discusión. La erupción polimórfica del embarazo es un trastorno inflamatorio benigno de la piel. Inicia con la aparición de pápulas pruriginosas que confluyen hasta formar placas eritematosas, que aparecen primero en el abdomen con excepción de la zona umbilical y parten, generalmente, de las estrías y se diseminan a las extremidades. El tratamiento consiste en la utilización de emolientes y antihistamínicos para el control de las lesiones y el prurito.

Conclusiones. Aunque se trata de una patología benigna y autolimitada, es importante llegar al diagnóstico correcto e iniciar un manejo médico adecuado ya que síntomas como el prurito pueden generar lesiones por rascado que suelen infectarse y comprometer el estado de salud de las pacientes.

Palabras clave:

Exantema; Enfermedades de la Piel; Periodo Posparto; Prurito; Mujeres Embarazadas; Anomalías Cutáneas.

\section{ABSTRACT}

Introduction. During pregnancy, at least $20 \%$ of the expectant mothers are affected by several forms of dermatosis, which involve a heterogeneous group of skin conditions with a variety of manifestations and evolution. The objective is to present a case of initial polymorphic eruption during the postpartum period with a brief review of the literature related to the pathology and its treatment.

Case report. We present the case of a 38 year-old postpartum patient in her first pregnancy, who exhibited a clinical case of skin eruption and pruritus that started in the abdominal area and extended to the lower limbs. The lesions consisted of erythematous papules that clustered together to form plaques. She was diagnosed with polymorphic eruption of pregnancy and was treated with antihistamines, which resolved the pathology.

Discussion. Polymorphic eruption of pregnancy is a benign inflammatory disorder of the skin It starts with the appearance of pruritic papules that cluster together to form erythematous plaques that initially appear in the abdomen (with the exception of the umbilical area) and generally start at the stretch marks and disseminate to the extremities. Treatment consists of the use of emollients and antihistamines to control the lesions and the pruritus.

Conclusions. Although this is a benign, self-limiting condition, it is important to reach a correct diagnosis and initiate adequate medical treatment, as symptoms like pruritus can generate lesions from scratching that usually become infected and compromise the patients' health.

Keywords:

Exanthem; Diseases of the Skin; Post-Partum Period; Pruritus; Pregnant Women; Skin Anomalies.

\section{RESUMO}

Introdução. Na gravidez, as dermatoses afetam menos de $20 \%$ das gestantes e representam um grupo heterogêneo de condições da pele, com uma forma variada de apresentação e evolução. O objetivo é apresentar um caso de erupção polimorfa que iniciou durante o puerpério, com uma breve revisão de literatura sobre a patologia e seu tratamento.

Relato de caso. Apresentamos o caso de uma paciente de 38 anos no puerpério de sua primeira gestação, quem desenvolveu um quadro clínico de erupção cutânea e prurido que iniciou na região abdominal e espalhou-se nos membros inferiores. As lesões estavam constituídas por pápulas eritematosas que confluíam até formar placas. 
Foi diagnosticada com erupção polimórfica da gravidez e recebeu anti-histamínicos, conseguindo a resolução da patologia.

Discussão. A erupção polimórfica da gravidez é uma doença inflamatória benigna da pele. Começa com o aparecimento de pápulas pruriginosas que confluem até formar placas eritematosas, que aparecem primeiro no abdômen com exceção da área umbilical e geralmente parte das estrias e se espalham para as extremidades. O tratamento consiste no uso de emolientes e anti-histamínicos para o controle das lesões e o prurido.

Conclusão. Ainda seja uma patologia benigna e autolimitada, é importante acertar o diagnóstico e iniciar o tratamento médico adequado, uma vez que sintomas como o prurido podem gerar arranhões que tendem a se infectar e comprometer o estado de saúde das pacientes.

Palavras-chave:

Exantema; Dermatopatias; Período Pós-parto; Prurido; Gestantes; Anormalidades da Pele.

\section{Introducción}

Las dermatosis son enfermedades que afectan la piel que no necesariamente tienen como mecanismo la inflación o la infección. En el embarazo, las dermatosis afectan a menos del $20 \%$ de las gestantes y representan un grupo heterogéneo de afecciones cutáneas, con una forma variada de presentación y evolución $(1,2)$. Las dermatosis específicas del embarazo aparecen de forma predominante hacia el segundo y tercer trimestre de gestación e, incluso, en el postparto inmediato. En la actualidad, pertenecen a este grupo de patologías el herpes gestacional o penfigoide gestacional (PG), la erupción polimorfa del embarazo (EPE) y la erupción atópica del embarazo, la cual incluye el eccema atópico del embarazo, el prurigo del embarazo y la foliculitis pruriginosa del embarazo (3).

La erupción polimórfica del embarazo es un trastorno inflamatorio benigno de la piel, descrito por primera vez en 1979 por el dermatólogo estadounidense Thomas Lawley (4). La EPE se conoce también como el síndrome de pápulas y placas urticariformes y pruriginosas del embarazo (PPUPE). Históricamente, se la ha llamado erupción toxémica del embarazo, prurigo del embarazo de aparición tardía, erupción toxémica del embarazo de Bourne y eritema tóxico del embarazo, sin embargo, estos últimos términos han caído en desuso con el paso de los años $(5,6)$.

La EPE es un trastorno cutáneo frecuente en el embarazo, a pesar de ello, es poco diagnosticado y reportado en la literatura médica mundial $(5,7)$. Su incidencia varía entre 1 en 160 y 1 en 300 embarazos por año. La mayoría de los casos se presentan en primigestantes durante el tercer trimestre, entre las semanas 35 a 39 e, incluso, hay casos reportados inmediatamente después del parto
$(8,9)$. No se conocen datos de incidencia en el territorio nacional. $(8,9)$

El objetivo de este trabajo es presentar un caso de erupción polimorfa del embarazo aparecido en el puerperio inmediato, describiendo a manera de revisión de la literatura los conocimientos que se tienen hasta la actualidad de dicha patología.

\section{Presentación del caso}

Primigesta de 38 años, fototipo de piel Fitzpatrick III, con embarazo de bajo riesgo, controles prenatales completos y ganancia de $12 \mathrm{~kg}$ durante la gestación. En el momento de la atención clínica: puerperio de 48 horas de evolución de parto vaginal eutócico sin complicaciones, con recién nacido a término de 39 semanas y 5 días, con buena adaptación neonatal. La paciente refiere que luego del alta hospitalaria, a las 6 horas inicia sensación pruriginosa en abdomen, la cual se extendió a los miembros inferiores, sin comprometer palmas ni plantas de los pies, con aparición súbita de pápulas eritematosas. A la exploración física las constantes vitales se encontraban dentro de los rangos normales, no había ictericia ni dolor abdominal. Se evidenció en piel de abdomen la presencia de pápulas eritematosas, confluentes, de borde bien definido y sobreelevado que respetaban la región periumbilical, con signos de grataje ungueal sobre estrías abdominales. Se observó un patrón de diseminación caudal hacia los miembros inferiores, especialmente en la porción anterior de los muslos y la región posterior de las piernas, respetando cara, palmas, plantas de los pies y mucosas (Figura 1). Por las características semiológicas de las lesiones y luego de realizar laboratorios que evidenciaron glutamato- 


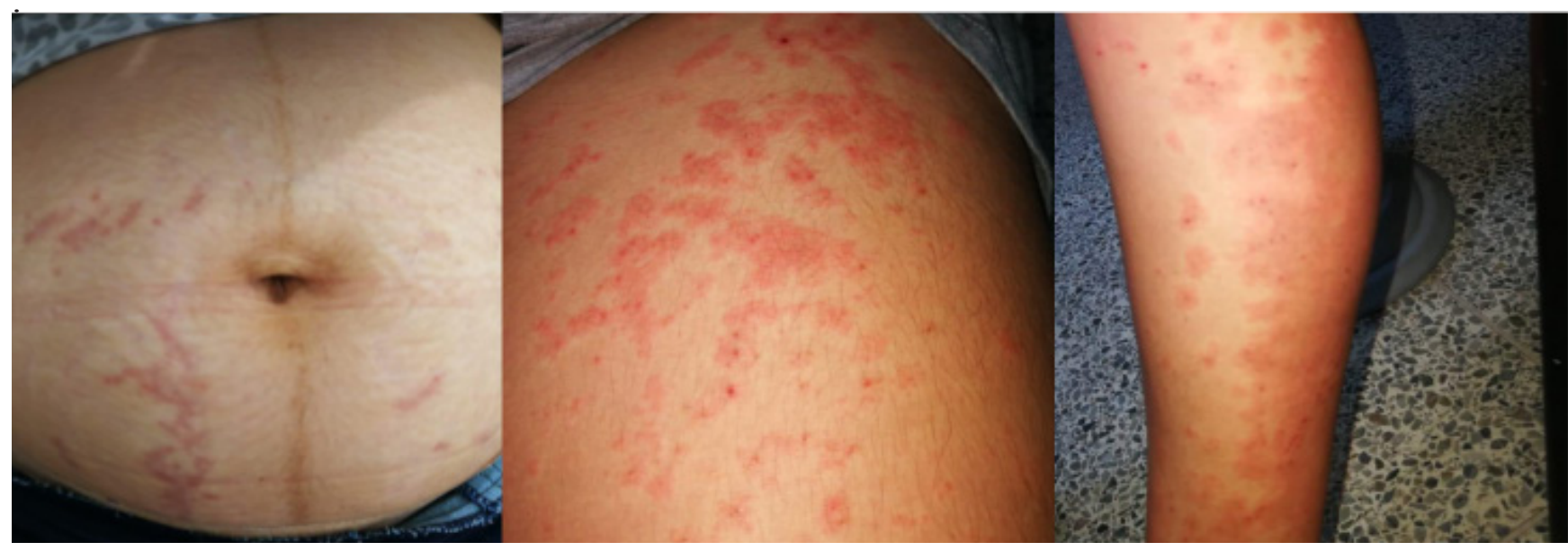

Figura 1. Fotografía de la paciente donde se evidencian lesiones sobre estrías, muslo y pierna izquierda, consistentes en rash maculopapular confluente, formando placas eritematosas con signos de grataje ungueal

Fuente: fotografía tomada por los autores.

piruvato transaminasa (GPT) en $15 \mathrm{U} / \mathrm{L}$, glutamatooxalacetato transaminasa (GOT) en $17 \mathrm{U} / \mathrm{L}$, fosfatasa alcalina en $50 \mathrm{U} / \mathrm{L}$, hemograma con hemoglobina de $11.5 \mathrm{~g} / \mathrm{dl}$, leucocitos de $6,000 \mathrm{~cm}^{3}$, creatinemia de 0.6 $\mathrm{mg} / \mathrm{dl}$, uroanálisis sin signos de infección ni proteinuria, test de Tzanck negativo, histopatología sin hallazgos de foliculitis e inmunofluorescencia de piel directa negativa para trastornos autoinmunes, se consideró el diagnóstico de erupción polimorfa del embarazo. Seguidamente, se inició tratamiento con antihistamínico (desloratadina 5 mg cada 24 horas), emolientes y uso de medios físicos, como colocación de compresas frías sobre las lesiones por 10 días. La paciente regresa a control 72 horas después del diagnóstico, con mejoría clínica del prurito y disminución de las lesiones, las cuales desaparecieron en su totalidad al cabo de 2 semanas.

\section{Discusión}

Las erupciones cutáneas constituyen un desafío diagnóstico para los médicos en atención primaria, especialmente aquellas que se presentan en mujeres gestantes o durante el puerperio. En la actualidad, son pocos los reportes presentes en la literatura médica mundial (1). Un estudio llevado a cabo por Panicker et al. en India, en 2016, contó con una muestra de 600 mujeres embarazadas con alteraciones cutáneas y reveló la presencia de erupción polimórfica en 8 pacientes, es decir, $1.3 \%$ del total de la muestra (10). En 2017, Kim describió el caso de una mujer de 34 años quien, luego de una semana del parto, presentó una erupción cutánea generalizada acompañada de prurito. Como dato adicional se observó una ganancia de peso durante la gestación de $13 \mathrm{~kg}$, situación similar a la reportada en el presente manuscrito (11). En cuanto al tratamiento del síndrome, han surgido nuevas alternativas como las reportadas por Jeon et al., donde tres pacientes rechazaron la medicación convencional por miedo a los efectos adversos, siendo manejadas con inyecciones intramusculares de sangre entera autóloga logrando la resolución de los síntomas (12). A continuación, se relatan aspectos importantes sobre la EPE.

En cuanto a los factores de riesgo principalmente asociados a este trastorno, sobresalen los relacionados con la sobredistensión del vientre materno, como el embarazo múltiple, el polihidramnios y la macrosomía fetal (situaciones que no estaban presentes en esta paciente). Algunos autores mencionan que, en los embarazos gemelares, la incidencia estimada es de 2.9 a $16 \%$ e incluso mayor en las gestaciones con más de tres fetos. También se conocen reportes donde se menciona un posible componente familiar, sin embargo, esta aseveración no cuenta con suficiente rigor científico que la avale $(3,5)$.

La fisiopatología de este trastorno no es muy clara. Se cree que el estiramiento de la piel rápidamente progresivo durante el tercer trimestre de la gestación genera la liberación de antígenos colágenos e, incluso, se menciona un posible estado de hiperreactividad inmune materna frente a estos antígenos, lo cual desencadenaría el cuadro clínico y explicaría la aparición de las lesiones sobre las estrías de la piel de las pacientes afectadas. Adicionalmente, se sabe que las células fetales o del 
trofoblasto que pasan a circulación materna estimulan la degranulación de los mastocitos; normalmente, los niveles de estrógenos y progesterona se incrementan y la progesterona empeora el proceso inflamatorio de la piel, por aumento en la inmunorreactividad del receptor de progesterona $(2,5)$.

Se han mencionado teorías sobre el papel de los antígenos paternos e, incluso, material masculino del feto como posible desencadenante de la patología, pero no se cuenta con la suficiente evidencia para determinar que se trata de una reacción de inmunidad o autoinmunidad. Los análisis de biopsias de piel revelan edema epidérmico y dérmico superior e infiltrado perivascular de neutrófilos, linfocitos $\mathrm{T}$, mastocitos y ocasionalmente eosinófilos $(11,13)$.
El cuadro clínico se caracteriza por la aparición de pápulas urticariformes que confluyen hasta formar placas eritematosas que se presentan en el área abdominal, respetando la zona umbilical y que, generalmente, parten de las estrías, diseminándose a extremidades superiores, glúteos y muslos, sin comprometer la cara, palmas ni plantas. La totalidad de estos hallazgos se encontraba presente en el caso de la paciente reseñada $(1,14)$.

Los principales diagnósticos diferenciales son otras condiciones pruriginosas asociadas al embarazo, como la erupción atópica del embarazo, el PG (también conocido como herpes gestacional) y la colestasis intrahepática del embarazo. Otras entidades patológicas que se pueden incluir en este grupo son la psoriasis pustulosa del embarazo, la urticaria, la dermatitis (atópica o por contacto) y los exantemas virales (5) (Figura 2) .

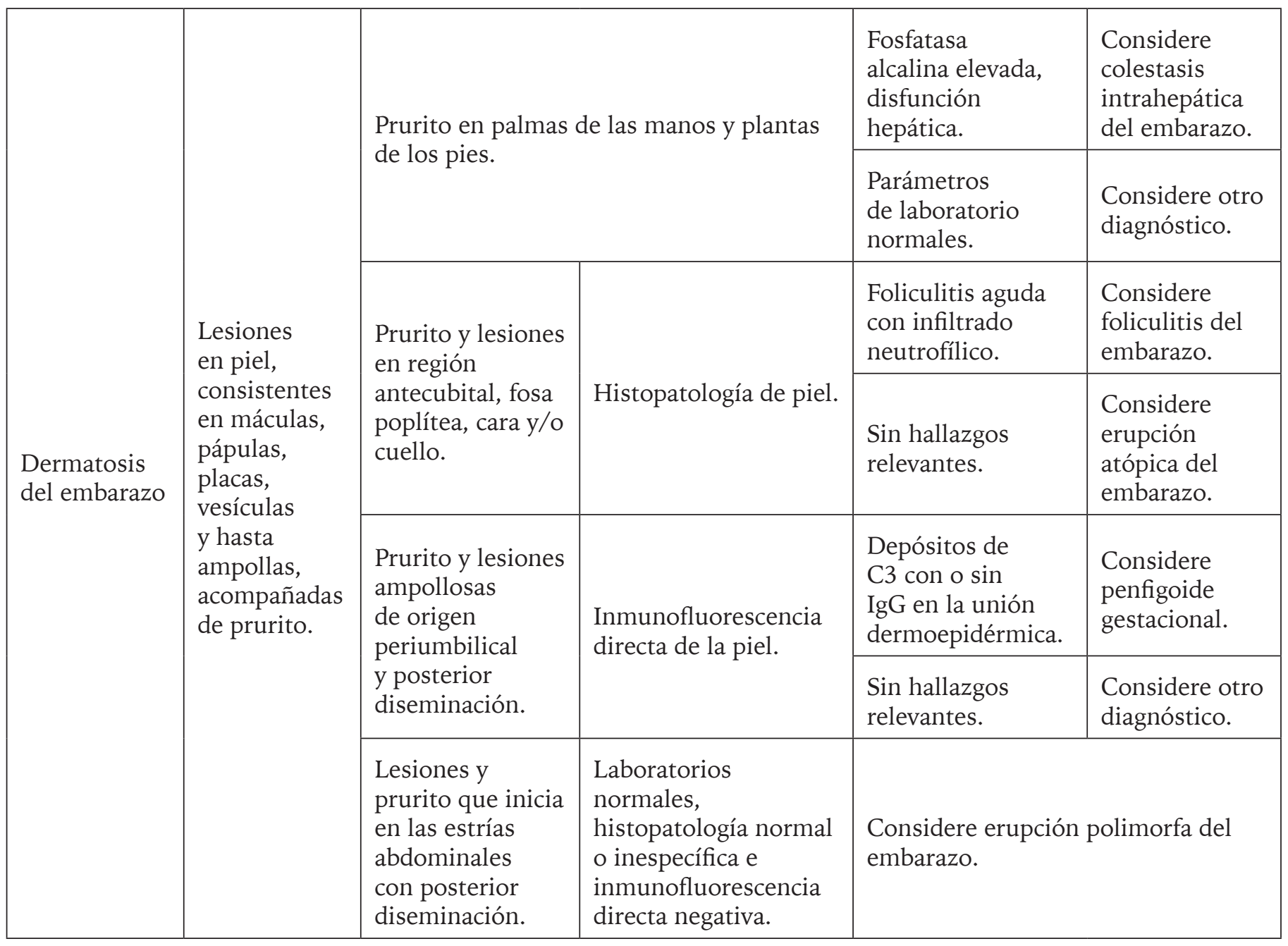

Figura 2. Flujograma de aproximación al diagnóstico diferencial de las dermatosis del embarazo.

Fuente: Elaboración propia de los autores. 
La erupción atópica del embarazo es una patología que incluye el eccema atópico del embarazo, el prurigo del embarazo y la foliculitis pruriginosa del embarazo. Tiene una aparición hacia el segundo trimestre de gestación y se caracteriza por la presencia de pápulas o nódulos excoriados, sin hallazgos histopatológicos o de laboratorio específicos, que aparecen en sitios típicos de reacciones atópicas, como la región antecubital, la fosa poplítea, cara, párpados y cuello: puntos muy diferentes a los sitios de aparición de la erupción polimórfica del embarazo $(5,15)$.

El PG es menos común. Aparece hacia el segundo trimestre, sus lesiones típicamente afectan el área umbilical, evitando las estrías y se describen como ampollas tensas. A pesar de las diferencias semiológicas de las lesiones, la erupción polimorfa puede ser difícil de distinguir de las ampollas del penfigoide gestacional. Debido a que el PG se asocia con un peor pronóstico fetal, con mayor incidencia de prematuridad y bajo peso al nacer, se debe realizar inmunofluorescencia directa, en la que se confirma el diagnóstico con la presencia de depósitos lineales de C3 a lo largo de la zona de membrana de la epidermis $(5,16)$.

Tanto la colestasis intrahepática (que representa un riesgo vital para la madre y el feto), como los exantemas virales, a menudo se presentan acompañados por otros signos y síntomas que inducen a sospechar su presencia $(17,18)$.

Por tratarse de una patología autolimitada de aproximadamente seis semanas de duración, sin consecuencias graves para la madre ni el feto, el tratamiento consiste en la utilización de emolientes y antihistamínicos para el control de las lesiones y el prurito. En casos más severos, con gran compromiso de la piel, se pueden necesitar ciclos cortos de corticoides orales. Los esteroides tópicos de baja potencia a potencia media son el tratamiento de primera línea, junto con emolientes blandos. Antihistamínicos orales, como la clorfeniramina y la difenhidramina, son medicamentos ideales para el prurito $(1,19)$.

Entre los antihistamínicos de segunda generación, la loratadina sigue siendo la primera opción, seguida por la cetirizina. El prurito intratable severo se trata con ciclos cortos de esteroides sistémicos o fototerapia con radiación ultravioleta $\mathrm{B}$. Se deben controlar los niveles de ácido fólico durante la fototerapia y se recomienda evitar las altas temperaturas. La prednisona es el esteroide sistémico preferido ya que no atraviesa la barrera feto-placentaria $(19,20)$. Tanto los corticoides como los antihistamínicos son medicamentos seguros durante la lactancia; sin embargo, se sugiere que, en caso de utilizar corticoides, se deben realizar ciclos cortos no mayores a 7 días para evitar supresión de la función adrenal en el neonato (20).

\section{Conclusiones}

El abordaje de las patologías relacionadas con la piel durante el embarazo y el puerperio es una labor desafiante para el médico. El prurito como síntoma acompaña muchas patologías significativas durante la gestación, y determinar su causa requiere un enfoque integral. El diagnóstico y tratamiento oportunos de enfermedades como la erupción polimorfa del embarazo ayudan a proporcionar una solución eficaz, reduciendo también la ansiedad de la paciente. Es crucial abordar y descartar los posibles diagnósticos diferenciales que varían en severidad según sus etiologías. (5)

Aunque la EPE se trata de una patología benigna y autolimitada, es importante disminuir síntomas como el prurito que pueden llevar a lesiones por grataje ungueal, que suelen infectarse y comprometer el estado de salud de las pacientes. $(1,9)$

\section{Consideraciones éticas}

Este manuscrito corresponde a una forma de publicación sin riesgo, ya que se basa en la descripción de un evento clínico, sin modificación de variables biológicas, sociales o geográficas. El texto se elaboró luego de la obtención del consentimiento informado por parte de la paciente, en el cual se especificó que los datos de identidad no serían revelados con la finalidad de mantener el anonimato.

\section{Conflictos de interés}

No existen conflictos de interés.

\section{Financiamiento}

Recursos propios de los autores.

\section{Agradecimientos}

A la clínica que participó en el desarrollo de la investigación con el aporte de la información correspondiente al caso. 


\section{Referencias}

1. Villanueva A, Sánchez I, Lin S. Síndrome de PPUPE: Pápulas y placas urticariformes y pruriginosas del embarazo. Rev Cent Dermatol Pascua [Internet]. 2016 25(2):62-5. Recuperado a partir de: http://www.medigraphic.com/pdfs/derma/cd-2016/cd162d.pdf

2. Gay-Muñoz P, López Padilla S. Erupción polimórfica del embarazo. Acta médica grup ángeles [Internet]. 2017; 15(2):146-7. Recuperado a partir de: http://www.medigraphic.com/pdfs/actmed/am-2017/ am172n.pdf

3. Acosta I, Valdés O, Valdés O, Coto A. Penfigoide gestacional una dermatosis específica del embarazo. Rev Cuba Med Intensiva y Emergencias [Internet]. 2016 ;15(3):1-6. Recuperado a partir de: http://www. medigraphic.com/pdfs/revcubmedinteme/cie-2016/ cie163f.pdf

4. Lawley T, Hertz K, Wade T, Ackerman B, Katz S. Pruritic urticarial papules and plaques of pregnancy. JAMA. 1979;241(16):1696-1699. doi: 10.1001/ jama.1979.03290420022018.

5. Brandão $P$, Sousa-Faria $B$, Marinho C, Vieira-Enes P, Melo A, Mota L. Polymorphic eruption of pregnancy: Review of literature. J Obstet Gynaecol (Lahore). 2017;37(2):137-140. doi: 10.1080/01443615.2016.1225019.

6. Ambros-Rudolph CM, Sticherling M. Spezifische Schwangerschaftsdermatosen. Hautarzt. 2017;68(2):87-94. doi: 10.1007/s00105-016-3922-z.

7. Sirikudta W, Silpa-Archa N. Polymorphic eruption of pregnancy presented with targetoid lesions: A report of two cases. Case Reports in Dermatology. 2013;5(2):138-43. doi: 10.1159/000351259.

8. Delorenze L, Fiszon L, Neves S, Guedes L, Batista W, Guedes E. Pruritic folliculitis of pregnancy. An Bras Dermatol. 2016;91(5 Suppl 1):66-8. doi: abd18064841.20164735.

9. Acosta R, Martínez L, Aldama A, Domínguez L, Celías L, Mendoza G. Penfigoide gestacional asociado a complicaciones en el neonato. Rev. Nac [Internet]. 2014;6(1):53-6. Recuperado a partir de: http:// scielo.iics.una.py/pdf/hn/v6n1/v6n1a07.pdf
10. Panicker V, Riyaz N, Balachandran P. A clinical study of cutaneous changes in pregnancy. J Epidemiol Glob Health. 2017:7:63-70. doi: 10.1016/j. jegh.2016.10.002.

11. Kim EH. Pruritic urticarial papules and plaques of pregnancy occurring postpartum treated with intramuscular injection of autologous whole blood. Case Rep Dermatol. 2017;9(1):151-156. doi: 10.1159/000473874.

12. Jeon IK, On HR, Oh SH, Hann SK. Three cases of pruritic urticarial papules and plaques of pregnancy (PUPPP) treated with intramuscular injection of autologous whole blood. J Eur Acad Dermatol Venereol. 2015;29(4):797-800. doi: 10.1111/jdv.12414.

13. Massone C, Cerroni L, Heidrun N, Brunasso A, Nunzi E, Gulia A, et al. Histopathological diagnosis of atopic eruption of pregnancy and polymorphic eruption of pregnancy: a study on 41 cases. Am J Dermatopathol. 2014;36(10):812-21. doi: 10.1097/ DAD.0000000000000067.

14. Taylor D, Pappo E, Aronson IK. Polymorphic eruption of pregnancy. Clin Dermatol. 2016;34(3):383-91. doi: 10.1016/j.clindermatol.2016.02.011.

15. Danesh M, Pomeranz MK, McMeniman E, Murase JE. Dermatoses of pregnancy: Nomenclature, misnomers, and myths. Clin Dermatol. 2016;34(3):314-9. doi: 10.1016/j.clindermatol.2016.02.002.

16. Vaughan Jones S, Ambros-Rudolph C, Nelson-Piercy C. Skin disease in pregnancy. BMJ. 2014;348(3):3489. doi: https://doi.org/10.1136/bmj.g3489.

17. Roth MM, Cristodor P, Kroumpouzos G. Prurigo, pruritic folliculitis, and atopic eruption of pregnancy: Facts and controversies. Clin Dermatol. 2016;34(3):392400. doi: 10.1016/j.clindermatol.2016.02.012.

18. Lambert J. Itch in pregnancy management. Curr Probl Dermatol. 2016;50:164-72. doi: 10.1159/000446087.

19. Kannambal K, Tharini GK. A screening study on dermatoses in pregnancy. J Clin Diagnostic Res. 2017;11(5):1-5.doi:10.7860/JCDR/2017/27207.9907.

20. Bechtel A. Pruritus in pregnancy and its management. Dermatol Clin. 2018;36(3):259-65. doi: 10.1016/j. det.2018.02.012. 